\title{
Nonlinear Tracking Control for Dual-Stage Actuator Systems
}

\author{
Jinchuan Zheng and Minyue Fu
}

\begin{abstract}
This paper presents a nonlinear control method for dual-stage actuator (DSA) systems to track a step command input fast and accurately. Conventional tracking controllers for DSA systems were generally designed to enable the primary actuator to approach the setpoint without overshoot. However, we observe that this strategy is unable to achieve the minimal settling time when the setpoints are beyond the secondary actuator travel limit. To further reduce the settling time, we design the primary actuator controller to yield a closedloop system with a small damping ratio for a fast rise time and certain allowable overshoot. Then, a composite nonlinear control law is designed for the secondary actuator to reduce the overshoot caused by the primary actuator as the system output approaches the setpoint. The proposed control method was applied to an actual DSA positioning system, which consists of a linear motor and a piezo actuator. Experimental results demonstrate that it can further reduce the settling time significantly compared with the conventional control.
\end{abstract}

\section{INTRODUCTION}

A dual-stage actuator (DSA) servo system is characterized by a structural design with two actuators connected in series along a common axis. The primary actuator (coarse actuator) is of long travel range but with poor accuracy and slow response time. The secondary actuator (fine actuator) is typically of higher precision and faster response but with a limited travel range. The two actuators are complementary to each other to provide both large travel range, high positioning accuracy and fast response. The DSA servomechanism has been widely used in the industry, e.g., [1]-[3].

Although the mechanical design of a DSA system appears to be simple, it is a challenging task to design controllers for the two actuators to yield an optimal performance. A variety of approaches have been reported to deal with the dual-stage control problems. For example, control design for track following and settling can be found in [4], [5]. The secondary actuator saturation problem was explicitly taken into account during the control design [6], [7]. In [8], a decoupled track-seeking controller using a three-step design approach is developed to enable high-speed one-track seeking and short-span track-seeking for a dual-stage servo system. The control design for the secondary actuator by minimizing the destructive interference is proposed in [9] to attain desired time and frequency responses.

In this paper, we consider a class of DSA systems that can be depicted by Fig. 1(a), where $M$ and $m$ represent the mass of the primary and secondary actuator, respectively. Fig. 1(b) shows an example of our developed DSA positioning system,

J. Zheng and M. Fu are with the School of Electrical Engineering and Computer Science, The University of Newcastle, Callaghan, NSW 2308, Australia. Jinchuan. Zheng @newcastle. edu. au; Minyue.Fu@newcastle.edu.au

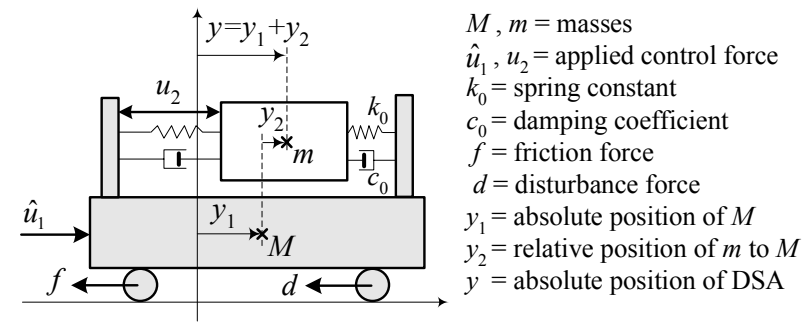

(a) Illustration of a DSA model

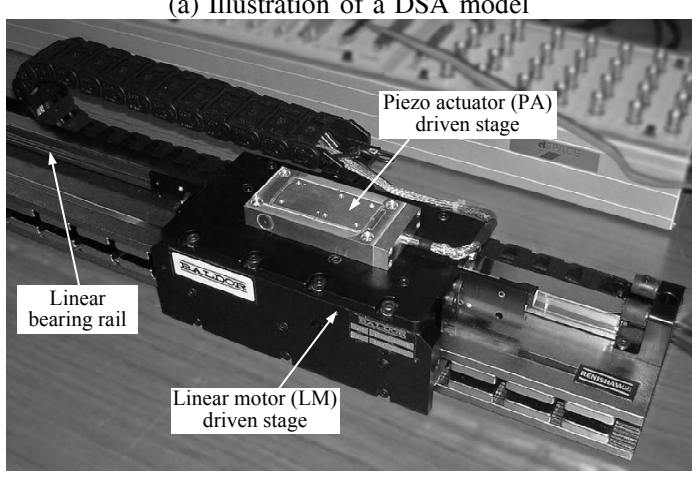

(b) A developed DSA positioning system

Fig. 1. DSA systems.

which consists of a primary stage driven by a linear motor (LM) and a secondary stage driven by a piezo actuator (PA). The secondary actuator has a limited travel range denoted by $\bar{y}_{2}$, which is very small relative to that of the primary actuator. Under the assumption of $M \gg m,\left|y_{1}\right| \gg\left|y_{2}\right|$, and $\left|\frac{u_{2}}{\hat{u}_{1}}\right| \gg \frac{m}{M}$, we can simply ignore the coupling forces between the two actuators and the dynamic equations of the DSA system are given by

$$
\left\{\begin{array}{l}
M \ddot{y}_{1}=\hat{u}_{1}-f\left(\dot{y}_{1}\right)-d \\
m \ddot{y}_{2}=u_{2}-c_{0} \dot{y}_{2}-k_{0} y_{2}
\end{array}\right.
$$

where the friction force $f\left(\dot{y}_{1}\right)$ is assumed to consist of Coulomb friction and viscous friction that can be described by the following equation:

$$
f\left(\dot{y}_{1}\right)=f_{c} \operatorname{sgn}\left(\dot{y}_{1}\right)+k_{v} \dot{y}_{1}
$$

where $f_{c}$ is the Coulomb friction level and $k_{v}$ is the viscous friction coefficient.

By far, most of the work on the DSA tracking control to follow a step command input is based on the strategy that the primary actuator control loop is designed to have little overshoot, and the secondary actuator control loop is designed to follow the position error of the primary actuator [8]-[10]. Under this conventional strategy, the total settling time can be reduced by the time that it takes for 
the secondary actuator to reach its travel limit. However, we observe that when the setpoint is beyond the secondary actuator travel range, this strategy is unable to minimize the total settling time because the secondary actuator can make little contributions due to its very limited travel range. To further reduce the settling time under this circumstance, we propose that the primary actuator controller can be designed to yield a closed-loop system with a small damping ratio for a fast rise time allowing a certain level of overshoot, and then as the primary actuator approaches the setpoint the secondary actuator control loop is used to reduce the overshoot caused by the primary actuator. In this way, the total settling time is obviously less than that of the conventional control provided that the overshoot caused by the primary actuator is within the secondary actuator travel range.

To perform the aforementioned control strategy, Section II presents a nonlinear tracking control method for the DSA systems in Fig. 1. Experimental results in Section III show that our proposed control can significantly speed up the step response compared with the conventional control.

\section{NONLINEAR TRACKING CONTROL DESIGN}

Our objective here is to design a control law such that the two actuators cooperate to enable the total position output $y$ to track a step command input of amplitude $y_{r}$ rapidly without exhibiting a large overshoot. In this section, we firstly present friction compensation for the primary actuator, and then a time-optimal control law is designed to yield a primary closed-loop system with a small damping ratio so as to achieve a quick rise time. Next, a composite nonlinear control law is designed for the secondary actuator to cause the DSA closed-loop system dynamics to be highly damped as the total position output approaches the setpoint, and thus the secondary actuator is enabled to reduce the overshoot caused by the primary actuator.

\section{A. Friction Compensation}

The nonlinear friction exerts adverse effect on the tracking performance. Generally, the friction parameters $f_{c}$ and $k_{v}$ in (2) can be estimated using the experimental method in [11]. Then, we can employ the model-based control structure as shown in Fig. 2 to compensate for the friction $f$ and disturbance $d$. The friction compensator is given by

$$
\begin{aligned}
u_{f} & =f_{c} \operatorname{sgn}\left(\dot{y}_{1}\right) \\
G_{n} & =\frac{1}{M s^{2}} \\
Q & =\frac{3 \tau s+1}{(\tau s)^{3}+3(\tau s)^{2}+3 \tau s+1}
\end{aligned}
$$

where $\tau$ is a time constant chosen as 5 to 10 times the servo bandwidth such that the filter $Q$ [12] can be approximated as $Q \approx 1$ within the frequency of interest. When the friction compensator is applied, the input-output relationship in Fig. 2 can be derived as

$$
y_{1}=\frac{u_{1}-(1-Q) d}{M s^{2}+(1-Q) k_{v} s} \approx \frac{1}{M s^{2}} u_{1} .
$$

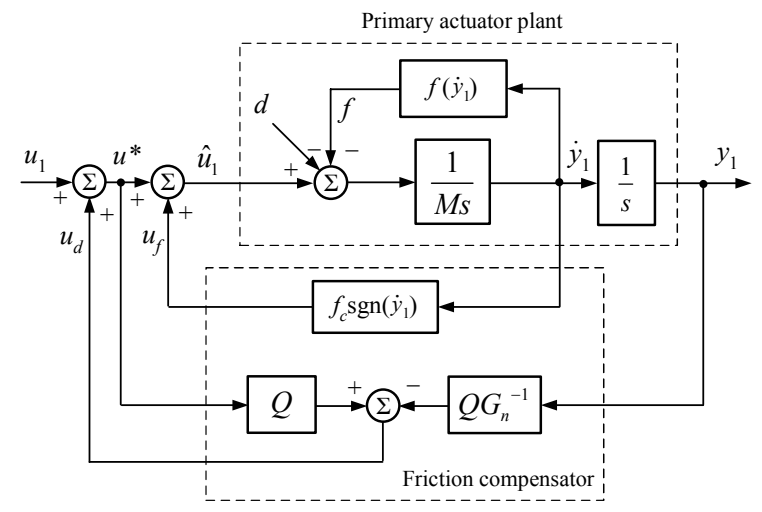

Fig. 2. Block diagram of friction compensation for the primary actuator.

It can be seen that the nonlinear friction and disturbance are approximately canceled by the friction compensator and the primary actuator system from $u_{1}$ to $y_{1}$ can be treated as a linear model with a pure double integrator, which facilitates the design of $u_{1}$ to further achieve desired performance.

From now on, we take (6) as the model of the primary actuator system and then rewrite the DSA model (1) in a state-space form as follows:

$$
\left\{\begin{aligned}
\Sigma_{1}: \dot{x}_{1} & =A_{1} x_{1}+B_{1} u_{1}, \quad x_{1}(0)=0 \\
\Sigma_{2}: \dot{x}_{2} & =A_{2} x_{2}+B_{2} \operatorname{sat}\left(u_{2}\right), \quad x_{2}(0)=0 \\
y & =y_{1}+y_{2}=C_{1} x_{1}+C_{2} x_{2}
\end{aligned}\right.
$$

where the state $x_{1}=\left[\begin{array}{ll}y_{1} & \dot{y}_{1}\end{array}\right]^{T}, x_{2}=\left[\begin{array}{ll}y_{2} & \dot{y}_{2}\end{array}\right]^{T}$, and

$$
\begin{aligned}
& A_{1}=\left[\begin{array}{ll}
0 & 1 \\
0 & 0
\end{array}\right], B_{1}=\left[\begin{array}{c}
0 \\
b_{1}
\end{array}\right], C_{1}=\left[\begin{array}{ll}
1 & 0
\end{array}\right], \\
& A_{2}=\left[\begin{array}{cc}
0 & 1 \\
a_{1} & a_{2}
\end{array}\right], B_{2}=\left[\begin{array}{c}
0 \\
b_{2}
\end{array}\right], C_{2}=\left[\begin{array}{ll}
1 & 0
\end{array}\right]
\end{aligned}
$$

with $b_{1}=\frac{1}{M}, a_{1}=-\frac{k_{0}}{m}, a_{2}=-\frac{c_{0}}{m}$ and $b_{2}=\frac{1}{m}$. It is clear that $A_{2}$ is Hurwitz and the travel limit of the secondary actuator is equivalently translated into input constraint with the saturation function $\operatorname{sat}\left(u_{2}\right)$ defined as

$$
\operatorname{sat}\left(u_{2}\right)=\operatorname{sgn}\left(u_{2}\right) \min \left\{\bar{u}_{2},\left|u_{2}\right|\right\}
$$

where $\bar{u}_{2}$ is the saturation level of the control input. Moreover, we assume that the states $x_{1}$ and $x_{2}$ are all measurable. Hence, the following control law for the DSA system (7) is based on full state feedback.

\section{B. Primary Actuator Control Design}

The role of the primary actuator is to provide large travel range beyond that of the secondary actuator. Thus, time optimal control is critical to move the position output quickly from one point to another. The proximate timeoptimal servomechanism (PTOS) is a practical near timeoptimal controller that can accommodate plant uncertainty and measurement noise. Hence, we apply the PTOS control law [13] to the primary actuator $\Sigma_{1}$ in (7) and the controller 
is independent of the secondary actuator control loop. The PTOS control law is given by

$$
\begin{aligned}
u_{1} & =\operatorname{sat}\left[k_{2}\left(f\left(e_{1}\right)-\dot{y}_{1}\right)\right] \\
f\left(e_{1}\right) & = \begin{cases}\frac{k_{1}}{k_{2}} e_{1} \\
\operatorname{sgn}\left(e_{1}\right)\left(\sqrt{2 \bar{u}_{1} b_{1} \alpha\left|e_{1}\right|}-\frac{\bar{u}_{1}}{k_{2}}\right) & \text { for }\left|e_{1}\right| \leq y_{l}>y_{l}\end{cases} \\
e_{1} & =y_{r}-y_{1}
\end{aligned}
$$

where sat[[-] is with the saturation level of $\bar{u}_{1}, \alpha$ is referred to as the acceleration discount factor, $k_{1}$ and $k_{2}$ are constant gains, and $y_{l}$ represents the size of a linear region. To make the functions $f\left(e_{1}\right)$ and $f^{\prime}\left(e_{1}\right)$ continuous such that the control input remains continuous as well, we have the following constraints

$$
\alpha=\frac{2 k_{1}}{b_{1} k_{2}^{2}}, \quad y_{l}=\frac{\bar{u}_{1}}{k_{1}} .
$$

The PTOS control law introduces a linear region close to the setpoint to reduce the control chatter. In the region $\left|e_{1}\right| \leq$ $y_{l}$, the control is linear and thus the gain $K=\left[\begin{array}{ll}k_{1} & k_{2}\end{array}\right]$ can be designed by any linear control techniques. For instance, using the pole-placement method we obtain a parameterized state feedback gain $K$ as follows

$$
K=\frac{1}{b_{1}}\left[\begin{array}{ll}
4 \pi^{2} \omega_{1}^{2} & 4 \pi \omega_{1} \zeta_{1}
\end{array}\right]
$$

where $\zeta_{1}$ and $\omega_{1}(\mathrm{~Hz})$ respectively represent the damping ratio and undamped natural frequency of the closed-loop system $C_{1}\left(s I-A_{1}+B_{1} K\right)^{-1} B_{1}$, whose poles are placed at $2 \pi \omega_{1}\left(-\zeta_{1} \pm j \sqrt{1-\zeta_{1}^{2}}\right)$.

In conventional DSA control systems, the primary actuator controller is generally designed to have little overshoot such as by choosing a large damping ratio in (13). However, in our proposed control a small damping ratio is chosen for a fast rise time and the resultant overshoot is within the secondary actuator travel limit, which can be then reduced by the secondary actuator under a composite nonlinear control law as will be given in Section II-C.

\section{Secondary Actuator Control Design}

The goal of the control design for the secondary actuator $\Sigma_{2}$ in (7) is to enable the secondary actuator to reduce the overshoot caused by the primary actuator. We have the following step-by-step design procedure.

Step 1: Design a linear feedback control law

$$
u_{2 L}=F x_{2}
$$

where $F=\left[\begin{array}{ll}f_{1} & f_{2}\end{array}\right]$ is chosen such that the secondary actuator control system as given by

$$
\dot{x}_{2}=A_{2} x_{2}+B_{2} \operatorname{sat}\left(F x_{2}\right)
$$

is globally asymptotically stable (GAS) and the corresponding closed-loop system in the absence of input saturation $C_{2}\left(s I-A_{2}-B_{2} F\right)^{-1} B_{2}$ has a larger damping ratio and a higher undamped natural frequency than those of the primary actuator control loop. To do this, we choose

$$
F=-B_{2}^{T} P
$$

where $P=P^{T}>0$ is the solution of the following Lyapunov equation

$$
A_{2}^{T} P+P A_{2}=-Q
$$

for a given $Q=Q^{T}>0$. Note that the solution of $P$ exists since $A_{2}$ is Hurwitz. To involve the closed-loop properties explicitly with the control law, we define

$$
Q=\left[\begin{array}{cc}
q_{1} & 0 \\
0 & q_{2}
\end{array}\right], q_{1}>0, q_{2}>0
$$

where $q_{1}$ and $q_{2}$ are tuning parameters. Substituting (18) into (17) yields $P$, which gives the feedback gain (16) as follows

$$
F=\frac{b_{2}}{2 a_{1} a_{2}}\left[a_{2} q_{1} \quad a_{1} q_{2}-q_{1}\right] .
$$

Moreover, the resulting poles of the closed-loop system $C_{2}\left(s I-A_{2}-B_{2} F\right)^{-1} B_{2}$ with (19) if complex conjugate have the undamped natural frequency and damping ratio as follows:

$$
\begin{aligned}
\omega_{2} & =\frac{1}{2 \pi} \sqrt{-\frac{b_{2}^{2}}{2 a_{1}} q_{1}-a_{1},} \\
\zeta_{2} & =\frac{b_{2}^{2} q_{1}-b_{2}^{2} a_{1} q_{2}-2 a_{1} a_{2}^{2}}{4 a_{1} a_{2} \sqrt{-\frac{b_{2}^{2}}{2 a_{1}} q_{1}-a_{1}}} .
\end{aligned}
$$

Thus, we can easily achieve the desired $\omega_{2}$ and $\zeta_{2}$ by choosing a proper pair of $q_{1}$ and $q_{2}$.

Step 2: Construct the nonlinear feedback control law

$$
\begin{aligned}
& u_{2 N}=\gamma\left(y_{r}, y\right) H\left[\begin{array}{c}
y_{1}-y_{r} \\
\dot{y}_{1}
\end{array}\right]
\end{aligned}
$$

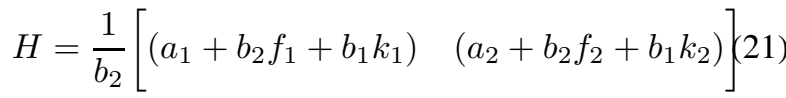

where $H$ is taken to achieve desired DSA closed-loop system dynamics, which will be clear in Section II-D; and $\gamma\left(y_{r}, y\right)$ is any nonnegative function locally Lipschitz in $y$, which is chosen to enable the secondary actuator to reduce the overshoot caused by the primary actuator as the total position output approaches the setpoint. The choice of $\gamma\left(y_{r}, y\right)$ will be discussed in Section II-D.

Step 3: Combine the linear and nonlinear feedback control laws derived in Steps 1 and 2 to form a composite nonlinear controller for the secondary actuator

$$
\begin{aligned}
u_{2} & =u_{1 L}+u_{2 N} \\
& =F x_{2}+\gamma\left(y_{r}, y\right) H\left[\begin{array}{c}
y_{1}-y_{r} \\
\dot{y}_{1}
\end{array}\right] .
\end{aligned}
$$

With the primary actuator controller in (9) and the secondary actuator controller as given by (22), we have the following results regarding the step response of the DSA closed-loop system.

Lemma 1: Consider the DSA system in (7) with the primary actuator $\Sigma_{1}$ under the PTOS control law (9) and the secondary actuator $\Sigma_{2}$ under the composite nonlinear control law (22) for any nonnegative function $\gamma\left(y_{r}, y\right)$ locally Lipschitz in $y$. Then the control laws will drive the total 
system output $y$ to track asymptotically any step command input of amplitude $y_{r}$.

Proof: The primary actuator closed-loop system under the PTOS control law can be represented as

$$
\dot{x}_{1}=A_{1} x_{1}+B_{1} \operatorname{sat}\left[k_{2}\left(f\left(e_{1}\right)-\dot{y}_{1}\right)\right]
$$

where $f\left(e_{1}\right)$ is defined in (10). It has been proved in [13] that the system (23) can track asymptotically any step command input of amplitude $y_{r}$, i.e.,

$$
\lim _{t \rightarrow \infty} y_{1}(t)=y_{r}, \quad \lim _{t \rightarrow \infty} \dot{y}_{1}(t)=0 .
$$

Next, we define a Lyapunov function $V=x_{2}^{T} P x_{2}$ with $P$ given in (17). Evaluating the derivative of $V$ along the trajectories of the system in (15) yields

$$
\begin{aligned}
\dot{V} & =\dot{x}_{2}^{T} P x_{2}+x_{2}^{T} P \dot{x}_{2} \\
& =x_{2}^{T}\left(A_{2}^{T} P+P A_{2}\right) x_{2}+2 B_{2}^{T} P x_{2} \operatorname{sat}\left(F x_{2}\right) \\
& =-x_{2}^{T} Q x_{2}-2 F x_{2} \operatorname{sat}\left(F x_{2}\right) \\
& \leq-x_{2}^{T} Q x_{2}<0 .
\end{aligned}
$$

Hence, the secondary actuator closed-loop system with the linear feedback control only (15) is GAS. Furthermore, the secondary actuator closed-loop system with the composite nonlinear control law (22) can be expressed as

$$
\dot{x}_{2}=A_{2} x_{2}+B_{2} \operatorname{sat}\left(F x_{2}+u_{2 N}\right) .
$$

It is obvious that the system (26) satisfies the converginginput bounded-state (CIBS) property (See [16] for the definition) since $A_{2}$ is Hurwitz, $|\operatorname{sat}(\cdot)| \leq \bar{u}_{2}$, and the nonlinear control input $u_{2 N}$ has

$$
\lim _{t \rightarrow \infty} u_{2 N}(t)=0
$$

which can be easily deduced from (20) and (24).

The proof finishes by observing that the DSA closed-loop system formed by (23) and (26) has a cascaded structure and it satisfies the conditions of Theorem 1 in [16]. It then follows that the cascade system formed by (23) and (26) is GAS at the origin. Thus, for the secondary actuator closedloop system (26) we have $\lim _{t \rightarrow \infty} x_{2}(t)=0$ and therefore,

$$
\lim _{t \rightarrow \infty} y(t)=\lim _{t \rightarrow \infty}\left[C_{1} x_{1}(t)+C_{2} x_{2}(t)\right]=y_{r} .
$$

Remark 1: Lemma 1 shows that the value of $\gamma\left(y_{r}, y\right)$ does not affect the ability of the overall DSA closed-loop system to track asymptotically any step command input. However, from the perspective of transient performance, a proper choice of $\gamma\left(y_{r}, y\right)$ should be carried out to improve the performance of the overall DSA closed-loop system. This is the key property of the proposed control design.

\section{Selecting $\gamma\left(y_{r}, y\right)$ for Improved Performance}

We design the primary actuator control loop with a small damping ratio for a quick rise time and employ the secondary actuator control loop that is designed to be highly damped to reduce the overshoot caused by the primary actuator as the total position output $y$ approaches the setpoint. This control strategy implies that the dynamics of the DSA closed-loop system should be dominated by the primary actuator control loop when the position output is far away from the setpoint, while dominated by the secondary actuator control loop when the position output approaches the setpoint. The purpose of the function $\gamma\left(y_{r}, y\right)$ is to fulfill a smooth transition from the primary control loop to the secondary control loop.

Consider the dual-stage system (7) with the control laws in (9) and (22), and assume that the tracking error $\left(y_{r}-y\right)$ is small such that the control inputs do not exceed the limits and the control law (9) works within its linear region. Thus, the DSA closed-loop system can be expressed as

$$
\Sigma:\left\{\begin{array}{l}
\dot{x}=A x+B y_{r} \\
y=C x
\end{array}\right.
$$

where

$$
\begin{aligned}
& A=\left[\begin{array}{cc}
A_{1}-B_{1} K & 0 \\
\gamma\left(y_{r}, y\right) B_{2} H & A_{2}+B_{2} F
\end{array}\right], \\
& B=\left[\begin{array}{c}
B_{1} K \\
-\gamma\left(y_{r}, y\right) B_{2} H
\end{array}\right] \cdot\left[\begin{array}{l}
1 \\
0
\end{array}\right], C=\left[\begin{array}{ll}
C_{1} & C_{2}
\end{array}\right] .
\end{aligned}
$$

The DSA closed-loop transfer function from $y_{r}$ to $y$ can be obtained by

$$
\begin{aligned}
G(s) & =C(s I-A)^{-1} B \\
& =(1-\gamma) G_{1}(s)+\gamma G_{2}(s)
\end{aligned}
$$

where

$$
\begin{aligned}
& G_{1}(s)=\frac{b_{1} k_{1}}{s^{2}+b_{1} k_{2} s+b_{1} k_{1}}, \\
& G_{2}(s)=\frac{-\left(a_{1}+b_{2} f_{1}\right)}{s^{2}-\left(a_{2}+b_{2} f_{2}\right) s-\left(a_{1}+b_{2} f_{1}\right)}
\end{aligned}
$$

are the closed-loop transfer function of the primary and secondary actuator control loop, respectively.

At this point, it is clear that the DSA closed-loop system dynamics (30) change from the primary control loop to the secondary control loop when $\gamma$ increases from 0 to 1 . This desired feature is due to the proper selection of $H$ in (21). From the perspective of zero placement, when $\gamma$ changes from 0 to 1 the zeros of (30) is moved from the pole locations of the secondary control loop (31) to those of the primary control loop (32). Since the zeros near the poles reduce the effects of the poles on the total response, we can use $\gamma$ to tune the system dynamics for desired performance. A similar control technique for SISO linear systems can be found in [14], which however uses the nonlinear feedback law to increase the damping ratio of the closed-loop system poles to reduce the overshoot.

The function $\gamma\left(y_{r}, y\right)$ can be chosen as a function of the tracking error. The following shows one choice of $\gamma$ :

$$
\gamma\left(y_{r}, y\right)=e^{-\beta\left|y_{r}-y\right|}
$$

where $\beta \geq 0$ is a tuning parameter. The function $\gamma\left(y_{r}, y\right)$ in (33) changes from 0 to 1 as $y \rightarrow y_{r}$. The parameter $\beta$ can be adjusted with respect to the amplitude of $y_{r}$ relative to the secondary actuator travel limit $\bar{y}_{2}$. 


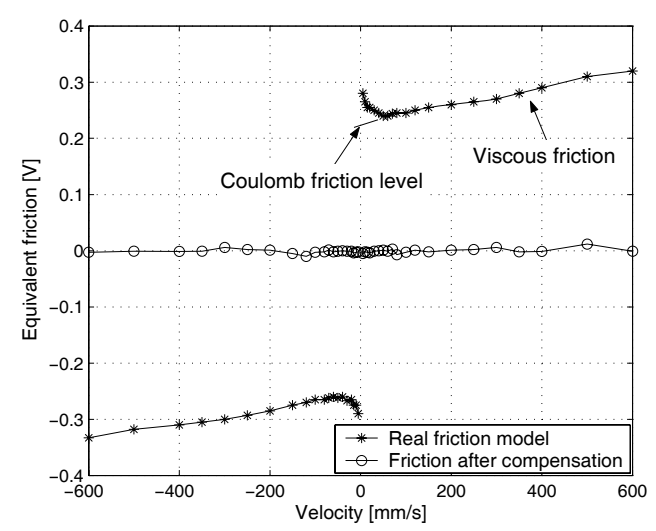

Fig. 3. Experimental friction model of the LM (The vertical axis denotes the steady-state control input $u_{1}$ in Fig. 2 that compensates for the friction force to make the LM move at the corresponding constant velocity).

\section{EXPERIMENTAL RESULTS}

This section presents the experimental results of the proposed nonlinear control method applied to the actual DSA positioning system as shown in Fig. 1(b).

\section{A. System Modeling}

The LM in Fig. 1(b) has a $0.5 \mathrm{~m}$ travel range, a $1 \mu \mathrm{m}$ resolution glass scale encoder, and a power amplifier. The PA has a maximum travel range of $\pm 15 \mu \mathrm{m}$, a piezoelectric amplifier, and an integrated capacitive position feedback sensor with $0.2 \mathrm{~nm}$ resolution to measure the relative displacement between the PA stage and the LM stage.

The nonlinear friction model in the LM is measured and shown in Fig. 3. The friction compensator in Fig. 2 is obtained by setting $f_{c}=0.22, \tau=0.0008$ and $M=$ $6.7 \times 10^{-8}$. It can be seen from Fig. 3 that the nonlinear friction is almost compensated by the friction compensator. Thus, the DSA positioning system can be expressed by (7), the parameters of which are then identified from experimental frequency response data as shown in Fig. 4. The LM dynamics is closed to a double integrator and the PA dynamics is of high stiffness that exhibits a flat gain in the low frequency range. By using the least square estimation method [15], we obtain the DSA model parameters in (7) as $b_{1}=1.5 \times 10^{7}, a_{1}=-10^{6}, a_{2}=-1810, b_{2}=3 \times 10^{6}, \bar{u}_{2}=$ $5 \mathrm{~V}$. The solid lines in Fig. 4 show that the identified models match the measured models well in the frequency range of interest.

\section{B. Results and Discussion}

We follow the proposed control design procedure to obtain the controller for the DSA positioning system. The PTOS controller in (9) for the LM is obtained by choosing $\bar{u}_{1}=$ $1 \mathrm{~V}, \omega_{1}=30 \mathrm{~Hz}$ and thus $y_{l}=422 \mu \mathrm{m}$. We find that $\zeta_{1}$ can be adjusted as

$$
\zeta_{1}\left(y_{r}\right)= \begin{cases}0.5, & y_{r} \leq 15 \mu \mathrm{m} \\ \frac{\ln \left(y_{r}\right)-2.7}{\sqrt{\pi^{2}+\left[\ln \left(y_{r}\right)-2.7\right]^{2}}}, & y_{r}>15 \mu \mathrm{m}\end{cases}
$$
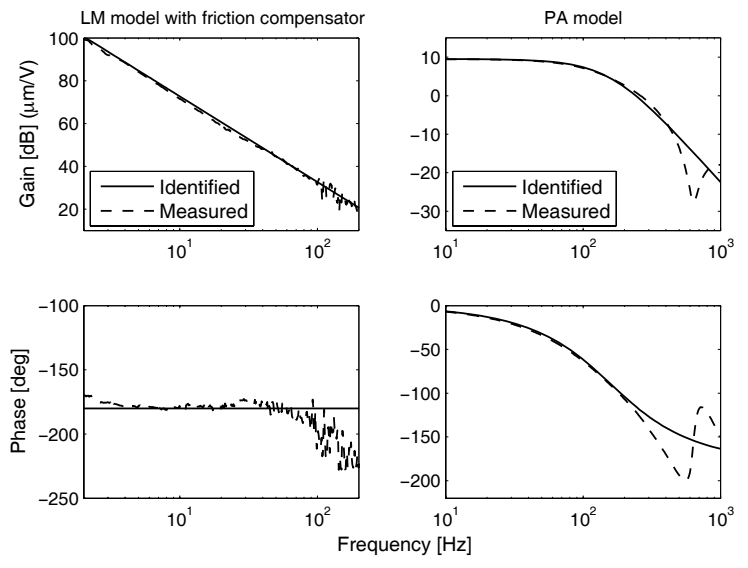

Fig. 4. Frequency responses of the LM model from $u_{1}$ to $y_{1}$ in Fig. 2 and the PA stage, the PA has a gain of $3 \mu \mathrm{m} / \mathrm{V}$.
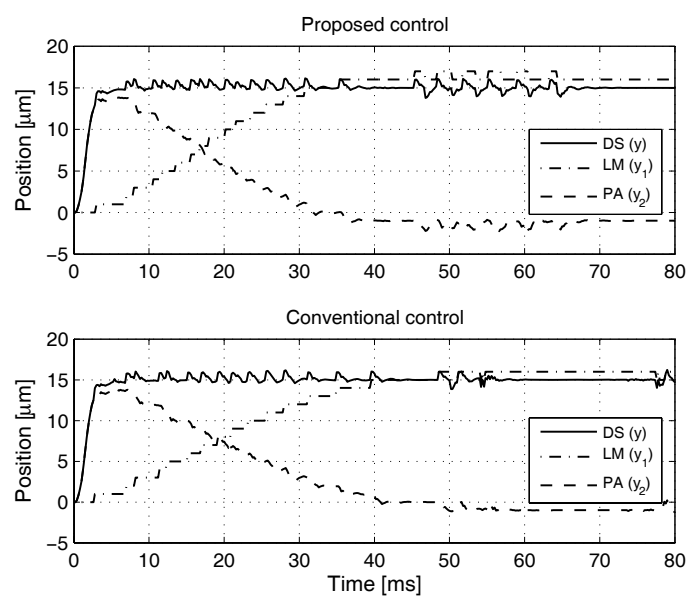

Fig. 5. Dual-stage tracking control for $y_{r}=15 \mu \mathrm{m}$. The settling times in both control are similarly $4 \mathrm{~ms}$. The proposed control has little improvement over the conventional control because within the PA travel limit the PA control loop dominates the DSA closed-loop system dynamics whatever the LM control loop is tuned.

such that the resultant overshoot caused by the LM approximately equals to the PA travel limit $(15 \mu \mathrm{m})$ when $y_{r}>15$ $\mu \mathrm{m}$. Hence, the linear gain $K$ is given by

$$
K=10^{-3} \times\left[\begin{array}{ll}
2.4 & 0.025 \zeta_{1}\left(y_{r}\right)
\end{array}\right]
$$

For the PA control design, we choose $q_{1}=0.56$ and $q_{2}=$ $7 \times 10^{-8}$ to obtain the linear feedback gain $F$ as follows

$$
F=-\left[\begin{array}{ll}
0.8385 & 0.0005
\end{array}\right]
$$

which results in $\omega_{2}=300 \mathrm{~Hz}$ and $\zeta_{2}=0.9$. The nonlinear feedback gain is given by

$$
H=-\left[1.1602 \quad 0.0011-0.00012 \zeta_{1}\left(y_{r}\right)\right]
$$

with $\zeta_{1}\left(y_{r}\right)$ in (34). The nonlinear function (33) is chosen as

$$
\gamma\left(y_{r}, y\right)= \begin{cases}e^{-0.001\left|y-y_{r}\right|}, & y_{r} \leq 15 \mu \mathrm{m} \\ e^{-0.01\left|y-y_{r}\right|} . & y_{r}>15 \mu \mathrm{m}\end{cases}
$$

In order to compare the proposed control with the conventional control where the LM control loop is generally tuned 

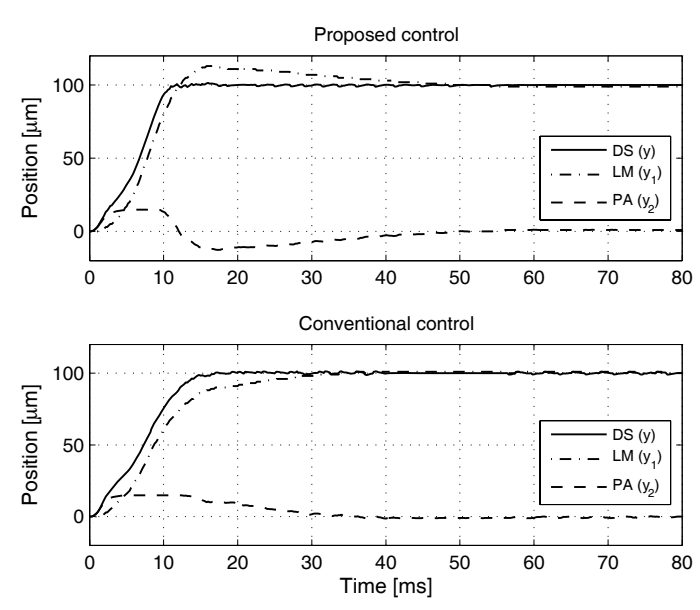

Fig. 6. Dual-stage tracking control for $y_{r}=100 \mu \mathrm{m}$. The settling time in the conventional control is $16.5 \mathrm{~ms}$, which is reduced to $11 \mathrm{~ms}$ in the proposed control.

TABLE I

COMPARISON OF THE SETTLING TIME IMPROVEMENT

\begin{tabular}{c|c|c|c}
\hline $\begin{array}{c}\text { Travel distance } \\
(\mu \mathrm{m})\end{array}$ & \multicolumn{2}{|c|}{ Settling Time (ms) } & Improvement \\
\cline { 2 - 3 } & Conventional & Proposed & $(\%)$ \\
\hline 15 & 4 & 4 & 0 \\
\hline 50 & 12.5 & 10 & 20 \\
\hline 100 & 16.5 & 11 & 33 \\
\hline 500 & 21 & 15.5 & 26 \\
\hline
\end{tabular}

to have no overshoot, we choose $\zeta_{1}=0.9$ for any $y_{r}$ and retain the other tuning parameters, then following the same design procedure yields a conventional controller that is used for comparison with our proposed controller.

The controllers were implemented by a real-time DSP system (dSPACE-DS1103) with the sampling frequency of 5 $\mathrm{kHz}$. The velocities of the LM and PA stage are estimated by the backward differentiation of the feedback position signals, respectively. The experimental results for $y_{r}=15,100 \mu \mathrm{m}$ are shown in Figs. 5 and 6. The results for some other travel distance are summarized in Table I for easy comparison. It is shown that the proposed control can further reduce the settling time by more than $20 \%$ when the travel distances are beyond $15 \mu \mathrm{m}$. Finally, we tested the performance of the proposed dual-stage control system under disturbance input. Fig. 7 shows that the proposed control can achieve improved positioning accuracy over the single-stage control.

\section{CONCLUSIONS AND FUTURE WORKS}

We have proposed a nonlinear control method for the DSA systems, where the primary actuator control loop has a small damping ratio for a fast rise time and the secondary actuator control loop is enabled by a nonlinear control law to reduce the overshoot caused by the primary actuator. We have also verified the proposed control on an actual DSA positioning system. Experimental results demonstrated that it can further reduce the settling time by more than $20 \%$ compared with the conventional control. Our future work will extend the nonlinear control design to higher order systems and the case of output feedback.
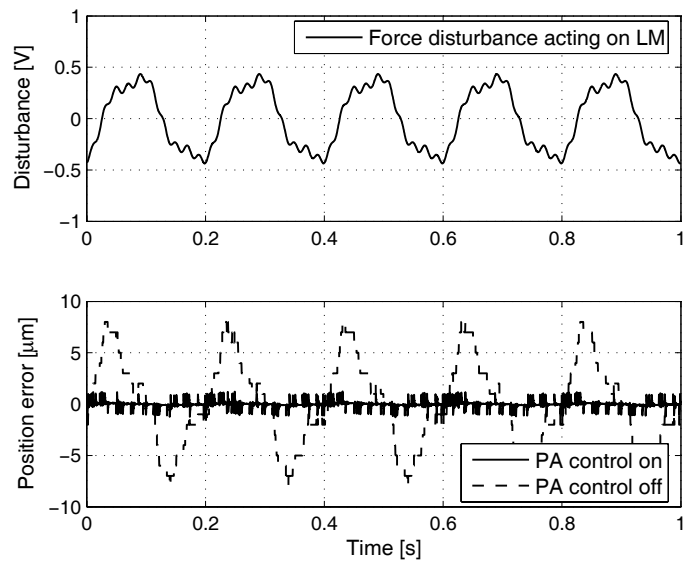

Fig. 7. Steady-state position error under disturbance input. When PA control is switched off, the position error under the LM control is within $\pm 8 \mu \mathrm{m}$, which is reduced to $\pm 1 \mu \mathrm{m}$ with PA control on.

\section{REFERENCES}

[1] R. Evans, J. Griesbach, and W. Messner, "Piezoelectric microactuator for dual stage control," IEEE Trans. Magn., vol. 35, no. 2, pp. 977-982, Mar. 1999.

[2] B. Kim, J. Li, and T. Tsao, "Two-parameter robust repetitive control with application to a novel dual-stage actuator for noncircular machining," IEEE/ASME Trans. Mechatron., vol. 9, no. 4, pp. 644-652, Dec. 2004.

[3] W. Yao, and M. Tomizuka, "Robust controller design for a dual-stage positioning system," in Proc. Int. Conf. Industrial Electronics, Control, and Instrumentation, 1993, pp. 62-66.

[4] S. Schroeck, W. Messner, and R. McNab, "On compensator design for linear time-invariant dual-input single-output systems," IEEE/ASME Trans. Mechatron., vol. 6, no. 1, pp. 50-57, Mar. 2001.

[5] H. Numasato and M. Tomizuka, "Settling control and performance of a dual-actuator system for hard disk drives," IEEE/ASME Trans. Mechatron., vol. 8, no. 4, pp. 431-438, Dec. 2003.

[6] G. Herrmann, M. Turner, I. Postlethwaite, and G. Guo, "Practical implementation of a novel anti-windup scheme in a HDD-dual-stage servo system," IEEE/ASME Trans. Mechatron., vol. 9, no. 3, pp. 580592, Sep. 2004.

[7] T. Shen, and M. Fu, "High precision and feedback control design for dual-actuator systems," in Proc. IEEE Conf. Control Applications, 2005, pp. 956-961.

[8] M. Kobayashi, and R. Horowitz, "Track seek control for hard disk dual-stage servo systems," IEEE Trans. Magn., vol. 37, no. 2, pp. 949-954, Mar. 2001.

[9] S. Lee, and Y. Kim, "Minimum destructive interference design of dualstage control systems for hard disk drives," IEEE Trans. Contr. Syst. Technol., vol. 12, no. 4, pp. 517-531, Jul. 2004.

[10] B. Hredzak, G. Herrmann, and G. Guo, "A proximate-time-optimal control design and its application to a hard disk drive dual-stage actuator system," IEEE Trans. Magn., vol. 42, no. 6, pp. 1708-1715, Jun. 2006.

[11] E. Papadopoulos, and G. Chasparis, "Analysis and model-based control of servomechanisms with friction," in Proc. IEEE/RSJ Int. Conf. Intelligent Robots and System, 2002, pp. 2109-2114.

[12] H. Choi, B. Kim, I. Suh, and W. Chung, "Design and robust highspeed motion controller for a plant with actuator saturation," Journal of Dynamic systems, Measurement, and Control, vol. 122, pp. 535-541, Sep. 2000.

[13] M. Workman, Adaptive Proximate Time-Optimal Servomechanisms, Ph.D. thesis, Stanford University, 1987.

[14] Z. Lin, M. Pachter, and S. Banda, "Toward improvement of tracking performance-nonlinear feedback for linear systems," Int. J. Control, vol. 70, pp. 1-11, 1998.

[15] L. Ljung, System Identification - Theory For the User, 2rd ed., PTR Prentice Hall, 1999.

[16] E. Sontag, "Remarks on stabilization and input-to-state stability," in Proc. IEEE Conf. Decision and Control, 1989, pp. 1376-1378. 